\title{
Serum microRNA profiles as prognostic or predictive markers in the multimodality treatment of patients with gastric cancer
}

\author{
LEILA SISIC ${ }^{1}$, DANIEL VALLBÖHMER ${ }^{2}$, NIKOLAS H. STOECKLEIN ${ }^{2}$, SUSANNE BLANK ${ }^{1}$, THOMAS SCHMIDT ${ }^{1}$, \\ CHRISTIANE DRIEMEL ${ }^{2}$, BIRTE MÖHLENDICK ${ }^{2}$, WOLFRAM T. KNOEFEL ${ }^{2}$, \\ MARGARETE ODENTHAL $^{3}$ and KATJA OTT $^{1}$ \\ ${ }^{1}$ Department of General, Visceral, Pediatric and Vascular Surgery, University of Heidelberg, Heidelberg D-69115; \\ ${ }^{2}$ Department of General, Visceral and Pediatric Surgery, University of Düsseldorf, Düsseldorf D-40225; \\ ${ }^{3}$ Institute of Pathology, University of Cologne, Cologne D-50441, Germany
}

Received June 15, 2014; Accepted February 27, 2015

DOI: $10.3892 / \mathrm{ol} .2015 .3341$

\begin{abstract}
Despite the implementation of multimodality treatment strategies, the persistently poor prognosis of gastric cancer patients is predominantly caused by the lack of predictive markers for response assessment in the neoadjuvant setting, preventing individualized therapy. Therefore, the identification of novel predictive and prognostic markers for application in the multimodality treatment of gastric cancer patients is required. The aim of the present study was to characterize the serum microRNA (miRNA/miR) profile of gastric cancer patients undergoing multimodality therapy to identify possible prognostic and predictive markers. The study consisted of 32 patients with gastric cancer who had undergone either primary surgical resection $(n=14)$ or neoadjuvant therapy followed by surgical resection $(n=18)$. Histopathological regression was defined as a major histopathological response when the resected specimens contained $<10 \%$ vital residual tumor cells. Intratumoral miRNA was isolated from pre-operative or post-neoadjuvant blood serum samples. Initially, microarray analyses were performed in six of the patients that received neoadjuvant treatment (three responders versus three non-responders), to assess the amplification profile of dysregulated miRNAs. Based on these findings, possible predictive or prognostic markers were validated in all study patients by performing single reverse transcription-polymerase chain reaction (RT-PCR) analysis. Depending on the extent of the histopathological regression, a differential miRNA expression profile was identified in the microarray analyses. Based on the amplification profile, miR-21, miR-29a and miR-221 were selected for additional
\end{abstract}

Correspondence to: Professor Daniel Vallböhmer, Department of General, Visceral and Pediatric Surgery, University of Düsseldorf, 5 Moore Street, Düsseldorf D-40225, Germany

E-mail: daniel.vallboehmer@med.uni-duesseldorf.de

Key words: gastric cancer, microRNAs, prognostic and predictive factors validation. However, the single RT-PCR measurements of the three selected miRNAs did not exhibit any prognostic or predictive value in the patients treated with primary resection or neoadjuvant therapy and resection. Thus, the current pilot study failed to identify a prognostic or predictive value in selected miRNAs using single RT-PCR measurements, however, the microarray results revealed a differential microRNA expression profile depending on the histopathological regression. The findings of the present study may have been affected by the small sample size.

\section{Introduction}

Gastric cancer is the second leading cause of cancer-related mortality worldwide, and the overall survival of patients with this tumor entity remains poor, as diagnosis frequently occurs at an advanced stage. $(1,2)$. Despite the implementation of multimodality treatment strategies for locally advanced tumors, patients with gastric cancer persistently exhibit a poor prognosis, possibly due to the continuing lack of effective prognostic clinicopathological factors and predictive markers for response assessment in the neoadjuvant setting, preventing individualization of therapy $(3,4)$. Therefore, predictive and prognostic markers in the multimodality therapy of gastric cancer are required.

microRNAs (miRNAs) are a class of endogenous, small non-coding RNAs that regulate gene expression by inhibiting mRNA translation (5). Recent studies have indicated that miRNAs are key in the carcinogenesis of solid tumors, including gastric cancer (6). In fact, Ueda et al (7) assessed the association between intratumoral miRNA expression and the progression and prognosis in the tissues samples of 353 gastric cancer patients. It was demonstrated that low expression levels of let-7 $\mathrm{g}$ and miR-433, as well as high expression levels of miR-214, were significantly associated with reduced overall survival independent of clinical covariates (7). In addition, by analyzing tumor tissue samples from 100 gastric cancer patients, Li et al (8) developed a seven-miRNA signature that was strongly associated with relapse-free and overall survival. Furthermore, Liu et al (9) investigated 68 patients with locally advanced gastric cancer 
undergoing neoadjuvant chemotherapy followed by surgical resection, and revealed that low levels of let-7i miRNA were significantly correlated with local invasion, lymphatic metastasis and a poor histopathological response. However, thus far, only a small number of studies have assessed whether miRNAs in the serum could serve as prognostic or predictive factors in the multimodality treatment of patients with gastric cancer (10-16). For example, Song et al (12) analyzed the expression levels of serum miR-21 in 103 gastric cancer patients and demonstrated that high levels of miR-21 expression were associated with an increased tumor size and an advanced pathological tumor stage, but not with patient prognosis (10).

Therefore, the aim of the present study was to characterize different miRNA profiles in the serum of three gastric cancer patients undergoing multimodality therapy based on histopathological response. In addition, the present study aimed to validate these miRNA profiles in a larger series of patients, including patients that had undergone primary resection and neoadjuvant therapy, to identify possible prognostic and predictive markers.

\section{Patients and methods}

Patients. The present retrospective translational study consisted of 32 patients with gastric cancer who underwent either primary surgical resection $(n=14)$ or neoadjuvant therapy followed by surgical resection $(n=18)$ at the Department of General, Visceral, Pediatric and Vascular Surgery, University of Heidelberg (Heidelberg, Germany) or the Department of General, Visceral and Pediatric Surgery, University of Düsseldorf (Düsseldorf, Germany) between January 2008 and December 2012. Patients with histologically confirmed gastric cancer, with no evidence of distant metastases who had or had not received neoadjuvant therapy were included in the study. Table I indicates the demographic and histopathological data of the patient cohort. Serum samples were used in accordance with the local policies of the Institutional Review Boards of the University of Heidelberg and the University of Düsseldorf.

Staging. Tumor-node-metastasis staging was performed according to the criteria of the International Union Against Cancer (17). Clinical staging consisted of endoscopy, endoscopic ultrasound in the majority of patients, a computed tomography (CT) scan of the thorax and abdomen, and positron emission tomography in a small number of selected cases that presented ambiguous CT results.

Therapeutic strategy. A total of 14 patients underwent primary surgical treatment, while 18 patients received neoadjuvant chemotherapy followed by surgical resection. In the cases treated with neoadjuvant chemotherapy, the following regimens were typically administered: Epirubicin $\left(50 \mathrm{mg} / \mathrm{m}^{2}\right)$, oxaliplatin $\left(100 \mathrm{mg} / \mathrm{m}^{2}\right)$ and capecitabine $\left(800 \mathrm{mg} / \mathrm{m}^{2}\right)$ or 5-fluorouracil $\left(2,600 \mathrm{mg} / \mathrm{m}^{2}\right)$, leucovorin $\left(200 \mathrm{mg} / \mathrm{m}^{2}\right)$, oxaliplatin $\left(85 \mathrm{mg} / \mathrm{m}^{2}\right)$ and docetaxel $\left(50 \mathrm{mg} / \mathrm{m}^{2}\right)$. Restaging was performed 2-3 weeks after the completion of neoadjuvant therapy. With regard to the surgical treatment, 14 patients underwent a subtotal gastrectomy, 16 patients underwent
Table I. Patient characteristics ${ }^{\mathrm{a}}$.

\begin{tabular}{|c|c|c|}
\hline Characteristic & $\mathrm{n}$ & $\%$ \\
\hline \multicolumn{3}{|l|}{ Gender } \\
\hline Male & 18 & 56 \\
\hline Female & 14 & 44 \\
\hline \multicolumn{3}{|l|}{ Neoadjuvant therapy } \\
\hline No & 14 & 44 \\
\hline Yes & 18 & 56 \\
\hline \multicolumn{3}{|l|}{ Type of surgical resection } \\
\hline Subtotal gastrectomy & 14 & 44 \\
\hline Total gastrectomy & 16 & 50 \\
\hline Transhiatal extended gastrectomy & 2 & 6 \\
\hline \multicolumn{3}{|l|}{$\mathrm{T}$ classification } \\
\hline урT1 & 5 & 16 \\
\hline ypT2 & 7 & 22 \\
\hline урT3/4 & 20 & 62 \\
\hline \multicolumn{3}{|l|}{ M classification } \\
\hline M0 & 32 & 100 \\
\hline M1 & 0 & 0 \\
\hline \multicolumn{3}{|l|}{ R classification ${ }^{b}$} \\
\hline R0 & 22 & 69 \\
\hline $\mathrm{R} 1$ & 8 & 25 \\
\hline \multicolumn{3}{|l|}{ Histopathological response } \\
\hline Minor & 14 & 44 \\
\hline Major & 4 & 12 \\
\hline
\end{tabular}

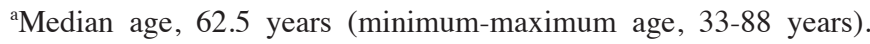
${ }^{b}$ Data of two patients incomplete. T, tumor; y, post-treatment; p, pathological; M, metastasis; R, resection.

a total gastrectomy and 2 patients underwent a transhiatal extended gastrectomy with D2-lymphadenectomy.

Histopathological grading of tumor response. An objective histopathological examination was performed to assess the extent of tumor regression in each case. The resected specimens were fixed in formalin (10\%), embedded in paraffin, cut into 5- $\mu \mathrm{m}$ slices, and stained with hematoxylin and eosin (Sigma-Aldrich, Munich, Germany). The prepared sections were analyzed to determine the effect of neoadjuvant therapy on the histopathological stage and regression. The number of vital residual tumor cells in the specimens was determined using a microscope. Specifically, histopathological regression was classified as a major histopathological response (i.e., responder) when $<10 \%$ vital residual tumor cells remained in the excised specimen. By contrast, a minor histopathological response (i.e., non-responder) was defined as a specimen containing $>10 \%$ vital residual tumor cells (18).

Serum samples and study design. Serum samples were collected from the 32 patients with gastric cancer prior to undergoing primary surgery or directly after completion of neoadjuvant therapy. Microarray-based analysis was employed to identify miRNAs that were differentially expressed between patients 
defined as histopathological responders and non-responders. This intratumoral miRNA profiling included a subset of six patient samples, including three patients exhibiting a major response and three patients exhibiting a minor response. Subsequently, the predictive values of the differentially-expressed miRNAs were assessed using reverse transcription-polymerase chain reaction (RT-PCR)-based analysis. RT-PCR analysis was performed on the serum samples of an additional 12 patients undergoing neoadjuvant therapy followed by surgical resection and all 14 patients undergoing primary surgical treatment. All methods are described below.

Total RNA isolation from serum samples. To allow subsequent normalization of extracellular miRNA levels, Simian virus (S V) 40-miRNA (2 pmol/200 $\mu \mathrm{l}$; Qiagen, Hilden, Germany) was added to the serum samples prior to RNA isolation. RNA was isolated from the serum samples using QIAzol lysis reagent, according to the manufacturer's instructions (Qiagen). RNA quantity was determined using a ND-1000 NanoDrop spectrophotometer at an absorbance of $260 \mathrm{~nm}$ (Thermo Fisher Scientific, Wilmington, DE, USA) and the quality of the RNA samples was assessed by performing microcapillary electrophoresis (2100 BioAnalyzer; Agilent Technologies, Inc., Waldbronn, Germany).

miRNA profiling using RT-PCR arrays. Serum RNA samples obtained from major and minor responders were subjected to miRNA profiling using a TaqMan ${ }^{\circledR}$ human microRNA array (version 2.0; Applied Biosystems Life Technologies, Carlsbad, CA, USA). RT of 300 ng total RNA from each sample was performed using Megaplex ${ }^{\mathrm{TM}}$ RT primers, human pools A and B, and a TaqMan miRNA RT kit (Applied Biosystems Life Technologies) to obtain complementary (c)DNA. The cDNA samples were loaded onto the microfluidic cards and miRNA profiling was performed by quantitative PCR (qPCR) array analysis using TaqMan Universal PCR Master Mix (Applied Biosystems Life Technologies) and the 7900HT fast real-time PCR system (Applied Biosystems Life Technologies). PCR conditions were as follows: Initial enzyme activation step at $95^{\circ} \mathrm{C}$ for $10 \mathrm{~min}$, followed by 45 cycles at $60^{\circ} \mathrm{C}$ for $1 \mathrm{~min}$ and $95^{\circ} \mathrm{C}$ for $20 \mathrm{sec}$. Sequence Detection System software (version 2.2; Applied Biosystems Life Technologies) was used to read the expression signals. Subsequently, these signals were normalized and interpreted by employing the $\Delta \Delta$ cycle threshold $(\mathrm{Ct})$ method, as well as cluster analysis. Ward's method and Manhattan distance interpretation were performed for this cluster analysis.

miRNA quantification by RT-PCR and subsequent qPCR. miRNA expression levels were analyzed by performing two-step qPCR using the miScript II RT kit and the miScript SYBR $^{\circledR}$ Green PCR kit (Qiagen). Primers for miR-21, miR-29b, miR-221 and SV-40 were used for cDNA synthesis, and the GeneGlobe search center (Qiagen) was used to identify appropriate primers for qPCR. PCR using $2 \mathrm{ng}$ cDNA was carried out in a $20 \mu \mathrm{l}$ assay under the following conditions: 45 cycles of $95^{\circ} \mathrm{C}$ for $15 \mathrm{sec}, 59^{\circ} \mathrm{C}$ for $30 \mathrm{sec}$ and $45^{\circ} \mathrm{C}$ for $45 \mathrm{sec}$. All experiments were performed in triplicate and in agreement with the manufacturer's instructions. Cellular miRNA levels were normalized using RNA U6 small nuclear 2 as the reference RNA. By contrast, spike-in SV40-miRNA (Qiagen) was used for normalization of extracellular miR-21, miR-29a and miR-221 levels.

Statistical analysis. Data were analyzed using non-parametric tests, including the Wilcoxon rank sum test for comparisons of paired data, the Kruskal-Wallis test for comparing greater than two groups and the Mann-Whitney test for comparing unpaired data. The maximal $\chi^{2}$ method, which was initially developed by Miller and Siegmund (19), and Halpern (20), was adapted to identify the miRNA expression value that resulted in optimal segregation of the cohort into poor and good prognosis groups (in terms of estimated survival time). In addition, the log-rank test used to determine the strength of the groupings. $\mathrm{P}<0.05$ was considered to indicate a statistically significant difference. Statistical analyses were performed using SPSS software for Windows (version 18.0; SPSS, Inc., Chicago, IL, USA). For cluster analysis and $\Delta \Delta \mathrm{Ct}$, RealTime StatMiner ${ }^{\circledR}$ software (Integromics, Granada, Spain) was used.

\section{Results}

miRNA profiles in the serum samples of gastric cancer patients depending on therapeutic response. Blood samples were obtained from 32 patients with gastric cancer. A total of 18 patients underwent neoadjuvant therapy followed by surgical resection and 14 patients underwent primary surgical resection. The blood samples were collected after neoadjuvant therapy prior to surgical resection or directly prior to primary surgery. Comprehensive miRNA profiling was performed using PCR-based microarray analyses from a subset of six pre-operatively treated patients (three patients exhibiting a major response and three patients exhibiting a minor response). The initial analysis assessed the differences between the miRNA profiles of the study patients and the miRNA profiles of healthy subjects. A cluster analysis was performed between the study patients and the healthy control subjects depending on the therapeutic response (no response versus response; Fig. 1). Furthermore, direct comparisons between the miRNA profiles of the responder and non-responder patients revealed that 112 miRNAs exhibited a divergent expression level of $>2.5$ between the two groups. In particular, miR-144*, miR-432*, miR-875-5p and miR-10b expression levels were decreased in the responders compared with the non-responders (Fig. 1).

Based on the microarray results, miR-432*, miR-144*, miR10b, miR-29a, miR-143, miR-192 and miR-221 exhibited differential expression between the responders and non-responders (Fig. 2). Therefore, these miRNAs were selected for additional validation in all study patients by single RT-PCR analyses. Quality control demonstrated that miR-432, miR-144, miR10b, miR-875-5p and miR-192 expression may be measured in a linear concentration-dependent manner in a limited detection range, while miR-29a and miR-221 exhibited an adequate detection range. In addition, analysis of the current literature was used to select miR-21 for additional validation analyses (13). Therefore, miR-21, miR-29b and miR-221 were selected for subsequent analysis.

miR-21, miR-29b and miR-221 expression following neoadjuvant therapy, and the association with histopathological response. miRNA expression levels in serum samples 


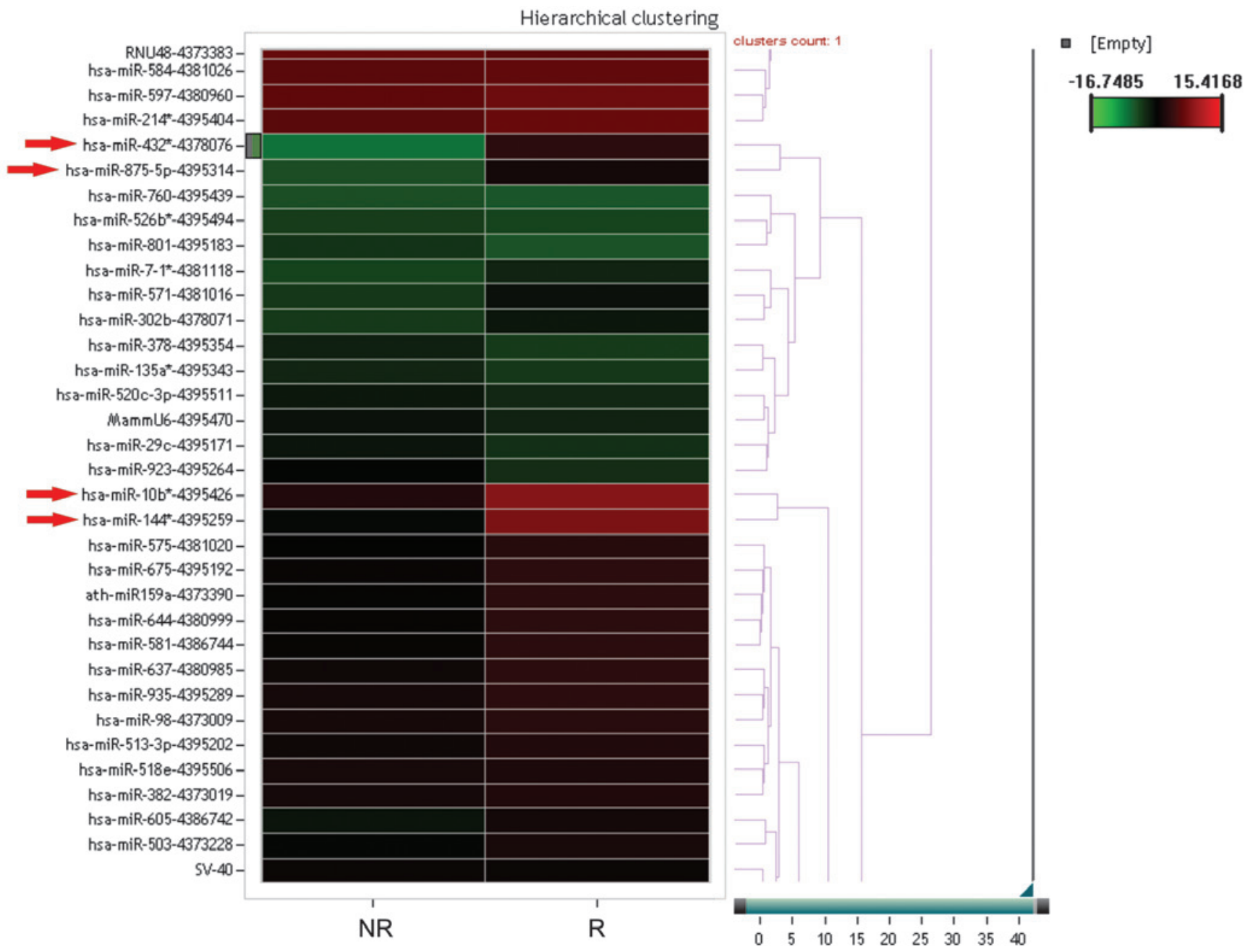

Figure 1. Cluster analysis of divergent miRNA profiles between study patients and healthy subjects following receipt of neoadjuvant therapy depending on the patient's histopathological response. Arrows indicate highly divergent expression of the miRNA species. R, responder; NR, non-responder; miRNA/miR, microRNA.

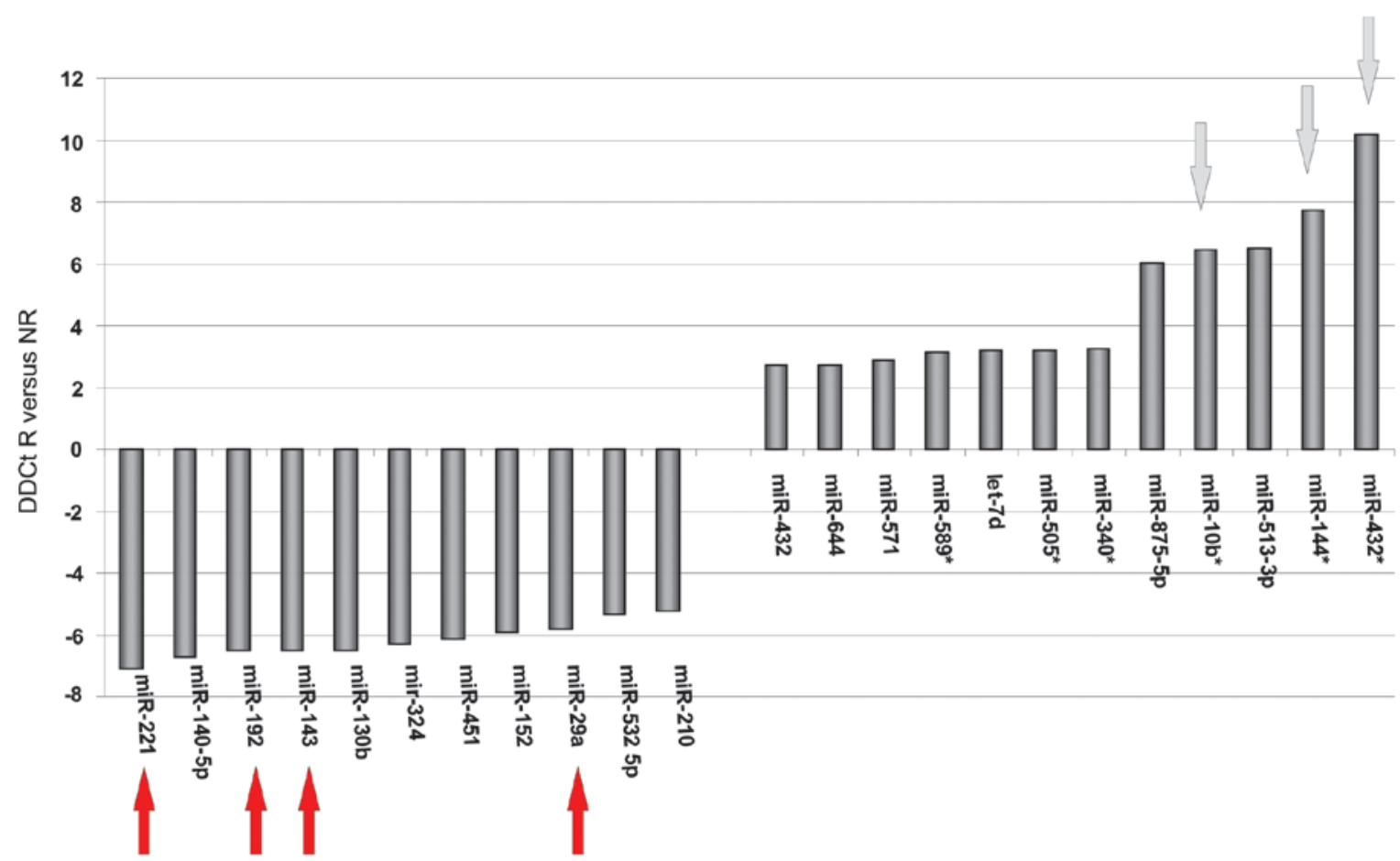

Figure 2. microRNA (miRNA/miR) expression profiles of gastric cancer patients with or without a histopathological response after neoadjuvant therapy (red arrows, miRNA increased in non-responders (NR) compared with responders (R); grey arrows, miRNA increased in Rs compared with NRs). DDCt, $\Delta \Delta$ cycle threshold. 
collected prior to primary surgical resection $(n=14)$ and after neoadjuvant therapy followed by surgical resection $(n=18)$ were compared. It was revealed that the neoadjuvant therapy did not significantly affect the expression of all the investigated miRNA and none of the selected miRNAs were significantly associated with histopathological response at any time-point ( $\mathrm{P}>0.05$; data not shown).

miR-21, miR-29b and miR-221 expression in serum samples of gastric cancer patients depending on clinicopathological factors. The miRNA expression analyses performed prior to neoadjuvant treatment as well as prior to direct surgical resection revealed that none of the three selected miRNAs significantly correlated with clinicopathological factors or survival ( $\mathrm{P}>0.05$; data not shown). Furthermore, no significant associations were detected when performing the analyses for all 32 study patients concurrently.

\section{Discussion}

The current translational pilot study aimed to identify possible prognostic and predictive markers in the multimodality treatment of gastric cancer. The miRNA profile was characterized in the serum of patients exhibiting gastric cancer who had undergone multimodality therapy or primary resection. The microarray analyses identified that a differential miRNA profile was expressed depending on the histopathological response of patients with locally advanced gastric cancer undergoing multimodality treatment. However, subsequent single RT-PCR analyses revealed no significant predictive or prognostic impact of the miRNAs in the two analyzed subgroups.

Methods for assessing prognosis and response are required to individualize multimodality therapy in gastric cancer, therefore, various studies have been conducted to characterize novel effective prognostic/predictive markers in this tumor entity $(21,22)$. For example, one of the largest studies, conducted by Ueda et al (7), analyzed tissue samples of 353 gastric cancer patients and identified that low expression levels of let-7 $\mathrm{g}$ and miR-433, as well as a high expression level of miR-214, were significantly associated with reduced overall survival independent of clinical covariates. However, the use of these possible markers is invasive, as an endoscopy is the most minimally invasive way to obtain an appropriate tissue biopsy for molecular analysis. Therefore, the identification of a marker in the peripheral blood (i.e., a non-invasive marker) would be valuable for effective prognostic and predictive assessment in the multimodality treatment of patients with gastric cancer.

In fact, recent studies investigating serum samples of gastric cancer patients revealed that miRNA expression can be detected in the blood and may have prognostic impact (10-16). However, to the best of our knowledge, thus far, no data exists regarding the predictive role of specific miRNAs in the serum of patients with gastric cancer.

By performing microarray analyses, the present study identified a differential miRNA expression profile depending on the histopathological response of patients with locally advanced gastric cancer undergoing multimodality treatment.
However, the current study failed to identify a significant predictive impact of these miRNAs in the single RT-PCR analyses. The following miRNAs were detected as possible predictive factors in the neoadjuvant treatment of gastric cancer followed by surgical resection: miR-432*, miR-144*, miR10b, miR-29a, miR-143, miR-192 and miR-221. Although these miRNAs have not previously been described as predictive factors in gastric cancer, miR-10b, miR-221, miR-29a and miR-192 in particular have been detected in other malignant tumors as possible response predictors (23-28). For example, Shen et al (23) demonstrated that the expression status of miR-21 and miR-10b in patients with non-small lung cancer was associated with disease progression, survival and response to adjuvant therapy with gefitinib. In addition, Preis et al (24) indicated that intratumoral miR-10b expression correlated with the response to neoadjuvant therapy and survival in patients with pancreatic ductal adenocarcinoma. Furthermore, evidence indicates that even miR-221 may serve as an effective factor for response prediction in human cancer. For example, Zhao et al (25) detected that plasma miR-221 expression may be a predictive biomarker for sensitivity to neoadjuvant chemotherapy in patients with breast cancer. This data is in accordance with a recent review conducted by Garofalo et al (26) regarding the role of miR-221 and miR-222 in tumor progression, and the response to antitumor therapy. The study described the two miRNAs as oncogenes or tumor suppressors that are dysregulated in different tumor entities and may serve a prognostic/predictive markers, as well as therapeutic tools, in cancer. In addition, Nagano et al (27) revealed that miR-29a induces resistance to gemcitabine in pancreatic cancer cells via the Wnt/ $\beta$-catenin signaling pathway. Finally, our previous study recently revealed that, in patients with locally advanced esophageal cancer undergoing neoadjuvant chemoradiation followed by esophagectomy, miR-192 and miR-194 levels in pre-therapeutic biopsies are considered to be indicators of a major histopathological response (28).

The present study may have several limitations. For example, it is a retrospective translational pilot study with a small number of study patients and therefore has the associated disadvantages. In addition, the patients in the present study were a heterogeneous group, as the cohort included patients that had and had not received neoadjuvant therapy. Other possible limitations of the present study include the time-point at which the blood samples were drawn.

In conclusion, the current pilot study did not identify significant prognostic or predictive value in the selected miRNAs upon single RT-PCR analysis, however, the microarray results revealed differential miRNA expression profiles depending on the extent of histopathological regression. Therefore, prospective translational studies with a high number of study patients are required to validate the results of the present study and to implement miRNAs as predictive factors in the multimodality treatment of patients with locally advanced gastric cancer.

\section{Acknowledgements}

The present study was funded by a grant from the Deutsche Forschungsgemeinschaft (no. VA506/3-1). 


\section{References}

1. Are C, Rajaram S, Are M, et al: A review of global cancer burden: trends, challenges, strategies and a role for surgeons. J Surg Oncol 107: 221-6, 2013.

2. Jemal A, Bray F, Center MM, Ferlay J, Ward E and Forman D: Global cancer statistics. CA Cancer J Clin 61: 69-90, 2011.

3. Cunningham D, Allum WH, Stenning SP, et al; MAGIC Trial Participants: Perioperative chemotherapy versus surgery alone for resectable gastroesophageal cancer. N Engl J Med 355: 11-20, 2006.

4. Ychou M, Boige V, Pignon JP, et al: Perioperative chemotherapy compared with surgery alone for resectable gastroesophageal adenocarcinoma: an FNCLCC and FFCD multicenter phase III trial. J Clin Oncol 29: 1715-21, 2011.

5. Ruan K, Fang X and Ouyang G: MicroRNAs: novel regulators in the hallmarks of human cancer. Cancer Lett 285: 116-126, 2009.

6. Wang F, Sun GP, Zou YF, Hao JQ, Zhong F and Ren WJ: MicroRNAs as promising biomarkers for gastric cancer. Cancer Biomark 11: 259-267, 2012.

7. Ueda T, Volinia S, Okumura H, et al: Relation between microRNA expression and progression and prognosis of gastric cancer: a microRNA expression analysis. Lancet Oncol 11: 136-146, 2010

8. Li X, Zhang Y, Zhang Y, Ding J, Wu K and Fan D: Survival prediction of gastric cancer by a seven-microRNA signature. Gut 59: 579-585, 2010.

9. Liu K, Qian T, Tang L, Wang J, Yang H and Ren J: Decreased expression of microRNA let-7i and its association with chemotherapeutic response in human gastric cancer. World J Surg Oncol 10: 225, 2012.

10. Liu R, Zhang C, Hu Z, et al: A five-microRNA signature identified from genome-wide serum microRNA expression profiling serves as a fingerprint for gastric cancer diagnosis. Eur J Cancer 47: 784-791, 2011.

11. Liu H, Zhu L, Liu B, et al: Genome-wide microRNA profiles identify miR-378 as a serum biomarker for early detection of gastric cancer. Cancer Lett 316: 196-203, 2012.

12. Song MY, Pan KF, Su HJ, et al: Identification of serum microRNAs as novel non-invasive biomarkers for early detection of gastric cancer. PLoS One 7: e33608, 2012.

13. Song J, Bai Z, Zhang J, et al: Serum microRNA-21 levels are related to tumor size in gastric cancer patients but cannot predict prognosis. Oncol Lett 6: 1733-1737, 2013.

14. Xu X, Yang X, Xing C, Zhang S and Cao J: miRNA: The nemesis of gastric cancer (Review). Oncol Lett 6: 631-641, 2013.

15. Tong F, Cao P, Yin Y, Xia S, Lai R and Liu S: MicroRNAs in gastric cancer: from benchtop to bedside. Dig Dis Sci 59: 24-30, 2014.
16. Wu $\mathrm{HH}$, Lin WC and Tsai KW: Advances in molecular biomarkers for gastric cancer: miRNAs as emerging novel cancer markers. Expert Rev Mol Med 16: e1, 2014.

17. Sobin LH, Gospodarowicz MK and Wittekind C (eds) TNM Classification of Malignant Tumours. 7th edition. Wiley-Blackwell, Oxford, 2009.

18. Becker K, Reim D, Novotny A, et al: Proposal for a multifactorial prognostic score that accurately classifies 3 groups of gastric carcinoma patients with different outcomes after neoadjuvant chemotherapy and surgery. Ann Surg 256: 1002-1007, 2012.

19. Miller R and Siegmund D: Maximally selected chi-square statistics. Biometrics 38: 1011-1016, 1982.

20. Halpern J: Maximally selected chi-square statistics for small samples. Biometrics 38: 1017-1023, 1982.

21. Yasui W, Oue N, Aung PP, Matsumura S, Shutoh M and Nakayama H: Molecular-pathological prognostic factors of gastric cancer: a review. Gastric Cancer 8: 86-94, 2005.

22. Pietrantonio F, De Braud F, Da Prat V, et al: A review on biomarkers for prediction of treatment outcome in gastric cancer. Anticancer Res 33: 1257-1266, 2013.

23. Shen Y, Tang D, Yao R, et al: microRNA expression profiles associated with survival, disease progression and response to gefitinib in completely resected non-small-cell lung cancer with EGFR mutation. Med Oncol 30: 750, 2013.

24. Preis M, Gardner TB, Gordon SR, et al: MicroRNA-10b expression correlates with response to neoadjuvant therapy and survival in pancreatic ductal adenocarcinoma. Clin Cancer Res 17: 5812-5821, 2011.

25. Zhao R, Wu J, Jia W, et al: Plasma miR-221 as a predictive biomarker for chemoresistance in breast cancer patients who previously received neoadjuvant chemotherapy. Onkologie 34: 675-680, 2011

26. Garofalo M, Quintavalle C, Romano G, Croce CM and Condorelli G: miR221/222 in cancer: their role in tumor progression and response to therapy. Curr Mol Med 12: 27-33, 2012.

27. Nagano H, Tomimaru Y, Eguchi H, et al: MicroRNA-29a induces resistance to gemcitabine through the Wnt/ $\beta$-catenin signaling pathway in pancreatic cancer cells. Int J Oncol 43: 1066-1072, 2013.

28. Odenthal M, Bollschweiler E, Grimminger PP, et al: MicroRNA profiling in locally advanced esophageal cancer indicates a high potential of miR-192 in prediction of multimodality therapy response. Int J Cancer 133: 2454-2463, 2013. 